\title{
CONCRETE SOLUTION TO THE NONSINGULAR QUARTIC BINARY MOMENT PROBLEM
}

\author{
RAÚL E. CURTO AND SEONGUK YOO \\ (Communicated by Pamela Gorkin)
}

\begin{abstract}
Given real numbers $\beta \equiv \beta^{(4)}: \beta_{00}, \beta_{10}, \beta_{01}, \beta_{20}, \beta_{11}, \beta_{02}, \beta_{30}$, $\beta_{21}, \beta_{12}, \beta_{03}, \beta_{40}, \beta_{31}, \beta_{22}, \beta_{13}, \beta_{04}$, with $\beta_{00}>0$, the quartic real moment problem for $\beta$ entails finding conditions for the existence of a positive Borel measure $\mu$, supported in $\mathbb{R}^{2}$, such that $\beta_{i j}=\int s^{i} t^{j} d \mu(0 \leq i+j \leq 4)$. Let $\mathcal{M}(2)$ be the $6 \times 6$ moment matrix for $\beta^{(4)}$, given by $\mathcal{M}(2)_{\mathbf{i}, \mathbf{j}}:=\beta_{\mathbf{i}+\mathbf{j}}$, where $\mathbf{i}, \mathbf{j} \in \mathbb{Z}_{+}^{2}$ and $|\mathbf{i}|,|\mathbf{j}| \leq 2$. In this note we find concrete representing measures for $\beta^{(4)}$ when $\mathcal{M}(2)$ is nonsingular; moreover, we prove that it is possible to ensure that one such representing measure is 6 -atomic.
\end{abstract}

\section{INTRODUCTION}

In this paper we find a direct proof that the nonsingular Quartic Binary Moment Problem always admits a finitely atomic representing measure with the minimum number of atoms, that is, six atoms. We do this in three steps:

(i) by normalizing the given moment matrix $\mathcal{M}(2)$ to ensure that $\mathcal{M}(1)$ is the identity matrix;

(ii) by developing a new rank-reduction tool, which allows us to decompose the normalized $\mathcal{M}(2)$ as the sum of a positive semidefinite moment matrix $\widetilde{\mathcal{M}(2)}$ of rank 5 and the rank-one moment matrix of the point mass at the origin; and

(iii) by proving that when a moment matrix $\mathcal{M}(2)$ admits such a decomposition, and $\widetilde{\mathcal{M}(2)}$ admits a column relation subordinate to a degenerate hyperbola (i.e., a pair of intersecting lines), then $\mathcal{M}(2)$ admits a 6 -atomic representing measure (as opposed to the expected 7-atomic measure).

To describe our results in detail, we need some notation and terminology. Given real numbers $\beta \equiv \beta^{(4)}: \beta_{00}, \beta_{10}, \beta_{01}, \beta_{20}, \beta_{11}, \beta_{02}, \beta_{30}, \beta_{21}, \beta_{12}, \beta_{03}, \beta_{40}, \beta_{31}, \beta_{22}$, $\beta_{13}, \beta_{04}$, with $\beta_{00}>0$, the Quartic Real Moment Problem for $\beta$ entails finding conditions for the existence of a positive Borel measure $\mu$, supported in $\mathbb{R}^{2}$, such that $\beta_{i j}=\int s^{i} t^{j} d \mu \quad(0 \leq i+j \leq 4)$. Let $\mathcal{M}(2)$ be the moment matrix for $\beta^{(4)}$,

Received by the editors May 12, 2014 and, in revised form, November 13, 2014 and December 13,2014 .

2010 Mathematics Subject Classification. Primary 47A57, 44A60, 42A70, 30A05; Secondary 15A15, 15-04, 47N40, 47A20.

Key words and phrases. Nonsingular quartic binary moment problem, moment matrix extension, flat extensions, rank-one perturbations, invariance under degree-one transformations.

The first named author was supported by NSF Grants DMS-0801168 and DMS-1302666. The second named author was supported by the PARC postdoctoral program at Seoul National University and by the Brain Korea 21 Program of National Research Foundation of Korea (Grant number: 22A20130012598). 
given by $\mathcal{M}(2)_{\mathbf{i}, \mathbf{j}}:=\beta_{\mathbf{i}+\mathbf{j}}$, where $\mathbf{i}, \mathbf{j} \in \mathbb{Z}_{+}^{2}$ and $|\mathbf{i}|,|\mathbf{j}| \leq 2$; this $6 \times 6$ matrix is shown below. (As is customary, the columns of $\mathcal{M}(2)$ are labeled $1, X, Y, X^{2}, X Y, Y^{2}$. In a similar way, given a collection of real numbers $\beta^{(2 n)}$ one defines the associated moment matrix by $\mathcal{M}(n)_{\mathbf{i}, \mathbf{j}}:=\beta_{\mathbf{i}+\mathbf{j}}$, where $\mathbf{i}, \mathbf{j} \in \mathbb{Z}_{+}^{2}$ and $|\mathbf{i}|,|\mathbf{j}| \leq n$.)

$$
\mathcal{M}(2) \equiv\left(\begin{array}{llllll}
\beta_{00} & \beta_{10} & \beta_{01} & \beta_{20} & \beta_{11} & \beta_{02} \\
\beta_{10} & \beta_{20} & \beta_{11} & \beta_{30} & \beta_{21} & \beta_{12} \\
\beta_{01} & \beta_{11} & \beta_{02} & \beta_{21} & \beta_{12} & \beta_{03} \\
\beta_{20} & \beta_{30} & \beta_{21} & \beta_{40} & \beta_{31} & \beta_{22} \\
\beta_{11} & \beta_{21} & \beta_{12} & \beta_{31} & \beta_{22} & \beta_{13} \\
\beta_{02} & \beta_{12} & \beta_{03} & \beta_{22} & \beta_{13} & \beta_{04}
\end{array}\right) .
$$

Assume now that $\mathcal{M}(2)$ is nonsingular. A straightforward consequence of Hilbert's Theorem yields the existence of a finitely atomic representing measure, as follows. Let $\mathcal{P}_{4}$ be the cone of nonnegative polynomials of degree at most 4 in $x, y$, regarded as a subset of $\mathbb{R}^{15}$. The dual cone is $\mathcal{P}_{4}^{*}:=\left\{\xi \in \mathbb{R}^{15}:\langle\xi, p\rangle \geq 0\right.$ for all $\left.p \in \mathcal{P}_{4}\right\}$. If $a, b \in \mathbb{R}$ and $\xi_{(a, b)}:=\left(1, a, b, a^{2}, a b, b^{2}, a^{3}, a^{2} b, a b^{2}, b^{3}, a^{4}, a^{3} b, a^{2} b^{2}, a b^{3}, b^{4}\right) \in \mathbb{R}^{15}$, then $\left\langle\xi_{(a, b)}, p\right\rangle=p(a, b) \geq 0$, for all $p \in \mathcal{P}_{4}$. Thus, $\xi_{(a, b)} \in \mathcal{P}_{4}^{*}$ for all $a, b \in \mathbb{R}$, and $\xi_{(a, b)}$ is also an extreme point. Consider now an arbitrary moment sequence $\beta^{(4)}$ with a nonsingular moment matrix $\mathcal{M}(2)$. Regarded as a point in $\mathbb{R}^{15}, \beta^{(4)}$ is in the interior of $\mathcal{P}_{4}^{*}$, since every $p \in \mathcal{P}_{4}$ is a sum of squares of polynomials. By the Krein-Milman Theorem and Carathéodory's Theorem, the Riesz functional $L_{\beta^{(4)}}$ (to be defined in Section 3) is a convex combination of evaluations $\xi_{(a, b)}$; that is, $\beta^{(4)}$ admits a finitely atomic representing measure, with at most 15 atoms. (In a recent related work, L.A. Fialkow and J. Nie FiNi obtained this result as a consequence of a more general result on moment problems.)

In this note we obtain a concrete 6 -atomic representing measure for $\mathcal{M}(2)$. The Quartic Real Binary Moment Problem admits an equivalent formulation in terms of complex numbers and representing measures supported in the complex plane $\mathbb{C}$, as follows. Given complex numbers $\gamma \equiv \gamma^{(4)}: \gamma_{00}, \gamma_{01}, \gamma_{10}, \gamma_{02}, \gamma_{11}, \gamma_{20}, \gamma_{03}, \gamma_{12}$, $\gamma_{21}, \gamma_{30}, \gamma_{04}, \gamma_{13}, \gamma_{22}, \gamma_{31}, \gamma_{40}$, with $\gamma_{i j}=\bar{\gamma}_{j i}$, one seeks necessary and sufficient conditions for the existence of a positive Borel measure $\mu$, supported in $\mathbb{C}$, such that

$$
\gamma_{i j}=\int \bar{z}^{i} z^{j} d \mu \quad(0 \leq i+j \leq 4)
$$

Just as in the real case, the Quartic Complex Moment Problem has an associated moment matrix $M(2)$, whose columns are conveniently labeled $1, Z, \bar{Z}, Z^{2}, \bar{Z} Z, \bar{Z}^{2}$. The most interesting case of the Singular Quartic Binary Moment Problem arises when the rank of $M(2)$ is 5 , and the sixth column of $M(2)$, labeled $\bar{Z}^{2}$, is a linear combination of the remaining five columns. Depending on the coefficients in the linear combination, four subcases arise in terms of the associated conic $C$ CuFi3, Section 5]: (i) $C$ is a parabola; (ii) $C$ is a nondegenerate hyperbola; (iii) $C$ is a pair of intersecting lines; and (iv) $C$ is a circle. In subcase (iii), it is possible to prove that the number of atoms in a representing measure (if it exists) may be 6 CuFi3, Proposition 5.5 and Example 5.6]; that is, in some soluble cases the rank of $M(2)$ may be strictly smaller than the number of atoms in any representing measure. 
Proposition 1.1 ([CuFi3, Proposition 5.5]). If $\mathcal{M}(2) \geq 0$, if $\operatorname{rank} \mathcal{M}(2)=5$, and if $X Y=0$ in the column space of $\mathcal{M}(2)$, then $\mathcal{M}(2)$ admits a representing measure $\mu$ with card $\operatorname{supp} \mu \leq 6$.

When combined with previous work on truncated moment problems, Proposition 1.1 led to the following solution to the truncated moment problem on planar curves of degree $\leq 2$. Given a moment matrix $\mathcal{M}(n)$ and a polynomial $p(x, y) \equiv \sum p_{i j} x^{i} y^{j}$, we let $p(X, Y):=\sum p_{i j} X^{i} Y^{j}$. A column relation in $\mathcal{M}(n)$ is therefore always described as $p(X, Y)=0$ for some polynomial $p$, with $\operatorname{deg} p \leq n$. We say that $\mathcal{M}(n)$ is recursively generated if for every $p$ with $p(X, Y)=0$ and every $q$ such that $\operatorname{deg} p q \leq n$ one has $(p q)(X, Y)=0$. In what follows, $v$ denotes the cardinality of the associated algebraic variety, defined as the intersection of the zero sets of all polynomials which describe the column relations in $\mathcal{M}(n)$.

Theorem 1.2 (CuFi4, Theorem 2.1], [Fia, Theorem 1.2]). Let $p \in \mathbb{R}[x, y]$, with $\operatorname{deg} p(x, y) \leq 2$. Then $\beta^{(2 n)}$ has a representing measure supported in the curve $p(x, y)=0$ if and only if $\mathcal{M}(n)$ has a column dependence relation $p(X, Y)=0$, $\mathcal{M}(n) \geq 0, \mathcal{M}(n)$ is recursively generated, and $r \leq v$.

The proof of Theorem 1.2 made use of affine planar transformations to reduce a generic quadratic column relation to one of four canonical types: $Y=X^{2}, X Y=1$, $X Y=0$, and $X^{2}+Y^{2}=1$; each of these cases required an independent result. We shall have occasion to use the affine planar transformation approach in Section 3 To date, most of the existing theory of truncated moment problems is founded on the presence of nontrivial column relations in the moment matrix $\mathcal{M}(n)$. On one hand, when all columns labeled by monomials of degree $n$ can be expressed as linear combinations of columns labeled by monomials of lower degree, the matrix $\mathcal{M}(n)$ is flat, and the moment problem has a unique representing measure, which is finitely atomic, with exactly $\operatorname{rank} \mathcal{M}(n-1)$ atoms [CuFi1, Theorem 1.1]. As a straightforward consequence, we conclude that for $n=1$, an invertible $\mathcal{M}(n)$ always admits a flat extension, while that is not the case for $n \geq 3$; that is, there exist examples of positive and invertible $\mathcal{M}(3)$ without a representing measure (cf. [CuFi1, Section 4]).

When $n=2$, the idea is to extend the $6 \times 6$ moment matrix $\mathcal{M}(2)$ to a bigger $10 \times 10$ moment matrix $\mathcal{M}(3)$ by adding so-called $B$ and $C$ blocks, as follows:

$$
\mathcal{M}(3) \equiv\left(\begin{array}{ll}
\mathcal{M}(2) & B(3) \\
B(3)^{*} & C(3)
\end{array}\right) .
$$

A result of J.L. Šmul'jan $[\mathrm{Smu}$ ] states that $\mathcal{M}(3) \geq 0$ if and only if (i) $\mathcal{M}(2) \geq 0$; (ii) $B(3)=\mathcal{M}(2) W$ for some $W$; and (iii) $C(3) \geq W^{*} \mathcal{M}(2) W$. Moreover, $\mathcal{M}(3)$ is a flat extension of $\mathcal{M}(2)$ (i.e., $\operatorname{rank} \mathcal{M}(3)=\operatorname{rank} \mathcal{M}(2)$ ) if and only if $C(3)=W^{*} \mathcal{M}(2) W$. Further, when $\mathcal{M}(2)$ is invertible, one easily obtains $W=$ $\mathcal{M}(2)^{-1} B(3)$, so in the flat extension case $C(3)$ can be written as $B(3)^{*} \mathcal{M}(2)^{-1} B(3)$. However, writing a general formula for $\mathcal{M}(3)$ is nontrivial, even with the aid of Mathematica, because of the complexity of $(\mathcal{M}(2))^{-1}$ and the new moments contributed by the block $B(3)$. On the other hand, if only one column relation is present (given by $p(X, Y)=0$ ), then $v=+\infty$, and the condition $r \leq v$, while necessary, will not suffice. One knows that the support of a representing measure must lie in the zero set of $p$, but this does not provide enough information to decipher the block $B(3)$. The situation is much more intriguing when no column relations are present; this is the nonsingular case, for which very little is known. 


\section{Statement of the main Result}

Theorem 2.1. Assume $\mathcal{M}(2)$ is positive and invertible. Then $\mathcal{M}(2)$ admits a representing measure, with exactly 6 atoms; that is, $\mathcal{M}(2)$ actually admits a flat extension $\mathcal{M}(3)$.

The proof of Theorem 2.1 is constructive, in that we first prove that it is always possible to switch from the invertible $\mathcal{M}(2)$ to a related singular matrix $\widetilde{\mathcal{M}(2)}$, with rank $\widetilde{\mathcal{M}(2)}=5$, for which Theorem 1.2 applies. Since singular positive semidefinite matrices $\mathcal{M}(2)$ always admit representing measures with 6 atoms or less, we can then conclude that an invertible positive $\mathcal{M}(2)$ admits a representing measure with at most 7 atoms. While this would already represent a significant improvement on the upper bound given by Carathéodory's Theorem (15 atoms), we have been able to establish that all positive invertible $\mathcal{M}(2)$ 's actually have flat extensions, and therefore their representing measures can have exactly 6 atoms.

\section{A NEW TOOL}

We begin this section with a result that will allow us to convert a given moment problem into a simpler, equivalent, moment problem. One of the consequences of this result is the equivalence of the real and complex moment problems, via the transformation $x:=\operatorname{Re}[z]$ and $y:=\operatorname{Im}[z]$; this equivalence has been exploited amply in the theory of truncated moment problems. For us, however, this simplification will allow us to assume that the submatrix $\mathcal{M}(1)$ is the identity matrix.

We adapt the notation in $\mathrm{CuFi3}$ ] to the real case. For $a, b, c, d, e, f \in \mathbb{R}$, $b f-c e \neq 0$, let $\Psi(x, y) \equiv\left(\Psi_{1}(x, y), \Psi_{2}(x, y)\right):=(a+b x+c y, d+e x+f y)(x, y \in$ $\mathbb{R})$. Given $\beta^{(2 n)}$, define $\tilde{\beta}^{(2 n)}$ by $\tilde{\beta}_{i j}:=L_{\beta}\left(\Psi_{1}^{i} \Psi_{2}^{j}\right)(0 \leq i+j \leq 2 n)$, where $L_{\beta}$ denotes the Riesz functional associated with $\beta$. (For $p(x, y) \equiv \sum p_{i j} x^{i} y^{j}$, the Riesz functional is given by $L_{\beta}(p):=p(\beta) \equiv \sum p_{i j} \beta_{i j}$.) It is straightforward to verify that $L_{\tilde{\beta}}(p)=L_{\beta}(p \circ \Psi)$ for every $p$ of degree at most $n$.

Proposition 3.1 (Invariance under degree-one transformations; CuFi3]). Let $\mathcal{M}(n)$ and $\tilde{\mathcal{M}}(n)$ be the moment matrices associated with $\beta$ and $\tilde{\beta}$, and let $J \hat{p}:=$ $\widehat{p \circ \Psi}$. Then the following statements hold.

(i) $\tilde{\mathcal{M}}(n)=J^{*} \mathcal{M}(n) J$.

(ii) $J$ is invertible.

(iii) $\tilde{\mathcal{M}}(n) \geq 0 \Leftrightarrow \mathcal{M}(n) \geq 0$.

(iv) $\operatorname{rank} \tilde{\mathcal{M}}(n)=\operatorname{rank} \mathcal{M}(n)$.

(v) $\mathcal{M}(n)$ admits a flat extension if and only if $\tilde{\mathcal{M}}(n)$ admits a flat extension.

We are now ready to put $\mathcal{M}(2)$ in "normalized form". Without loss of generality, we always assume that $\beta_{00}=1$. Let $d_{i}$ denote the leading principal minors of $\mathcal{M}(2)$; in particular,

$$
\begin{aligned}
& d_{2}=-\beta_{10}^{2}+\beta_{20}, \\
& d_{3}=-\beta_{02} \beta_{10}^{2}+2 \beta_{01} \beta_{10} \beta_{11}-\beta_{11}^{2}-\beta_{01}^{2} \beta_{20}+\beta_{02} \beta_{20} .
\end{aligned}
$$

Consider now the degree-one transformation

$$
\Psi(x, y) \equiv(a+b x+c y, d+e x+f y),
$$


where $a:=\frac{\beta_{01} \beta_{20}-\beta_{10} \beta_{11}}{\sqrt{d_{2} d_{3}}}, b:=\frac{\beta_{11}-\beta_{01} \beta_{10}}{\sqrt{d_{2} d_{3}}}, c:=-\sqrt{\frac{d_{2}}{d_{3}}}, d:=-\frac{\beta_{10}}{\sqrt{d_{2}}}, e:=\frac{1}{\sqrt{d_{2}}}$, and $f:=0$. Note that $b f-c e=-\sqrt{\frac{1}{d_{3}}} \neq 0$. Using this transformation, and a straightforward calculation, we can prove that any positive definite moment matrix $\mathcal{M}(2)$ can be transformed into the moment matrix

$$
\left(\begin{array}{cccccc}
1 & 0 & 0 & 1 & 0 & 1 \\
0 & 1 & 0 & \tilde{\beta}_{30} & \tilde{\beta}_{21} & \tilde{\beta}_{12} \\
0 & 0 & 1 & \tilde{\beta}_{21} & \tilde{\beta}_{12} & \tilde{\beta}_{03} \\
1 & \tilde{\beta}_{30} & \tilde{\beta}_{21} & \tilde{\beta}_{40} & \tilde{\beta}_{31} & \tilde{\beta}_{22} \\
0 & \tilde{\beta}_{21} & \tilde{\beta}_{12} & \tilde{\beta}_{31} & \tilde{\beta}_{22} & \tilde{\beta}_{13} \\
1 & \tilde{\beta}_{12} & \tilde{\beta}_{03} & \tilde{\beta}_{22} & \tilde{\beta}_{13} & \tilde{\beta}_{04}
\end{array}\right) .
$$

Thus, without loss of generality, we can always assume that $\mathcal{M}(1)$ is the identity matrix. We will now introduce a new tool in the study of moment matrices: the decomposition of an invertible $\mathcal{M}(2)$ as a sum of a moment matrix of rank 5 and a rank-one moment matrix.

Assume now that $\mathcal{M}(2)$ is invertible and that the submatrix $\mathcal{M}(1)$ is the identity matrix. For $u \in \mathbb{R}$ decompose $\mathcal{M}(2)$ as follows:

$$
\mathcal{M}(2)=\left(\begin{array}{cccccc}
1-u & 0 & 0 & 1 & 0 & 1 \\
0 & 1 & 0 & \beta_{30} & \beta_{21} & \beta_{12} \\
0 & 0 & 1 & \beta_{21} & \beta_{12} & \beta_{03} \\
1 & \beta_{30} & \beta_{21} & \beta_{40} & \beta_{31} & \beta_{22} \\
0 & \beta_{21} & \beta_{12} & \beta_{31} & \beta_{22} & \beta_{13} \\
1 & \beta_{12} & \beta_{03} & \beta_{22} & \beta_{13} & \beta_{04}
\end{array}\right)+\left(\begin{array}{cccccc}
u & 0 & 0 & 0 & 0 & 0 \\
0 & 0 & 0 & 0 & 0 & 0 \\
0 & 0 & 0 & 0 & 0 & 0 \\
0 & 0 & 0 & 0 & 0 & 0 \\
0 & 0 & 0 & 0 & 0 & 0 \\
0 & 0 & 0 & 0 & 0 & 0
\end{array}\right) .
$$

Denote the first summand by $\widehat{\mathcal{M}(2)}$ and the second summand by $\mathcal{P}$. It is clear that $\mathcal{P}$ is positive semidefinite and has rank 1 if and only if $u>0$, and in that case $\mathcal{P}$ is the moment matrix of the 1 -atomic measure $u \delta_{(0,0)}$, where $\delta_{(0,0)}$ is the point mass at the origin.

Proposition 3.2. Let $\mathcal{M}(2), \widehat{\mathcal{M}(2)}$, and $\mathcal{P}$ be as above, and let $u_{0}:=\frac{\operatorname{det} \mathcal{M}(2)}{R_{11}}$, where $R_{11}$ is the $(1,1)$ entry in the positive matrix $R:=(\mathcal{M}(2))^{-1}$. Then, with this nonnegative value of $u$, we have (i) $\widehat{\mathcal{M}(2)} \geq 0$; (ii) $\operatorname{rank} \widehat{\mathcal{M}(2)}=5$; and (iii) $\widehat{\mathcal{M}(2)}$ is recursively generated. Moreover, $u_{0}$ is the only value of $u$ for which $\widehat{\mathcal{M}(2)}$ satisfies (i)-(iii).

For the proof of Proposition 3.2 we will need the following auxiliary result, which is an easy consequence of the multilinearity of the determinant.

Lemma 3.3. Let $M$ be an $n \times n$ invertible matrix of real numbers, let $E_{11}$ be the rank-one matrix with $(1,1)$-entry equal to 1 and all other entries are equal to zero, and let $u \in \mathbb{R}$. Then $\operatorname{det}\left(M-u E_{11}\right)=\operatorname{det} M-u \operatorname{det} M_{\{2,3, \cdots, n\}}$, where $M_{\{2,3, \cdots, n\}}$ denotes the $(n-1) \times(n-1)$ compression of $M$ to the last $n-1$ rows and columns. In particular, if $u=\frac{\operatorname{det} M}{\left(M^{-1}\right)_{11}}$, then $\operatorname{det}\left(M-u E_{11}\right)=0$.

Proof of Proposition 3.2. (ii) Observe that $6=\operatorname{rank} \mathcal{M}(2) \leq \operatorname{rank} \widehat{\mathcal{M}(2)}+\operatorname{rank} \mathcal{P}=$ $\operatorname{rank} \widehat{\mathcal{M}(2)}+1$, so rank $\widehat{\mathcal{M}(2)} \geq 5$. Since $\operatorname{det} \widehat{\mathcal{M}(2)}=0$, we have rank $\widehat{\mathcal{M}(2)}=5$.

(i) Using the Nested Determinant Test starting at the lower right-hand corner of $\widehat{\mathcal{M}(2)}$, we know that $\widehat{\mathcal{M}(2)}$ is positive semidefinite since the nested determinants 
corresponding to principal minors of size 1, 2, 3, 4, and 5 are all positive, and the rank of $\widehat{\mathcal{M}(2)}$ is 5 . This also implies that $1-u \geq 0$. We now claim that $1-u$ is strictly positive. If $1-u=0$, then the positive semidefiniteness of $\widehat{\mathcal{M}(2)}$ would force all entries in the first row to be zero. Since this is evidently false, we conclude that $1-u>0$.

(iii) It is sufficient to show that the first three columns of $\widehat{\mathcal{M}(2)}$ are linearly independent. Consider the third leading principal minor of $\widehat{\mathcal{M}(2)}$, which equals $1-$ $u$, and is therefore positive. Thus, there is no linear dependence in this submatrix, and as a result the same holds in $\widehat{\mathcal{M}(2)}$.

Finally, the uniqueness of $u_{0}$ as the only value satisfying (i)-(iii) is clear.

\section{Proof of the MAIN Result}

We first observe that by combining Proposition 3.2 with Theorem 1.2 it suffices to consider the case when $\widehat{\mathcal{M}(2)}$ has a column relation corresponding to a pair of intersecting lines. For, in all other cases, there exists a representing measure for $\widehat{\mathcal{M}(2)}$ with exactly five atoms; when combined with the additional atom coming from the matrix $\mathcal{P}$, we see that $\mathcal{M}(2)$ admits a 6 -atomic representing measure.

We thus focus on the case when $\widehat{\mathcal{M}(2)}$ is subordinate to a degenerate hyperbola. After applying an additional degree-one transformation, we can assume, as in Proposition 1.1 that the column relation $X Y=0$ is present in $\widehat{\mathcal{M}(2)}$. However, we may not continue to assume that the submatrix $\widehat{\mathcal{M}(1)}$ is the identity matrix, since the degree-one transformation that produces the column relation $X Y=0$ will, in general, change the low-order moments. That is, $\widehat{\mathcal{M}(2)}$ is of the form

$$
\widehat{\mathcal{M}(2)}=\left(\begin{array}{cccccc}
1 & a & b & c & 0 & d \\
a & c & 0 & e & 0 & 0 \\
b & 0 & d & 0 & 0 & f \\
c & e & 0 & g & 0 & 0 \\
0 & 0 & 0 & 0 & 0 & 0 \\
d & 0 & f & 0 & 0 & h
\end{array}\right) .
$$

In this case, the original moment matrix $\mathcal{M}(2)$ is written as

$$
\mathcal{M}(2)=\widehat{\mathcal{M}(2)}+u\left(\begin{array}{lllllllllll}
1 & p & q & p^{2} & p q & q^{2}
\end{array}\right)^{T}\left(\begin{array}{llllll}
1 & p & q & p^{2} & p q & q^{2}
\end{array}\right),
$$

for some $u>0$ and $p q \neq 0$; that is, $\mathcal{M}(2)$ is the sum of a moment matrix of rank 5 with column relation $X Y=0$ and a positive scalar multiple of the moment matrix associated with the point mass at $(p, q)$, with $p q \neq 0$. Without loss of generality, we can assume that $p=q=1$ (this requires an obvious degree-one transformation, i.e., $\left.\tilde{x}:=\frac{x}{p}, \tilde{y}:=\frac{y}{q}\right)$. As a result, the form of $\mathcal{M}(2)$ is now as follows:

$$
\mathcal{M}(2)=\left(\begin{array}{cccccc}
1+u & a+u & b+u & c+u & u & d+u \\
a+u & c+u & u & e+u & u & u \\
b+u & u & d+u & u & u & f+u \\
c+u & e+u & u & g+u & u & u \\
u & u & u & u & u & u \\
d+u & u & f+u & u & u & h+u
\end{array}\right) .
$$


We will show that $\mathcal{M}(2)$ admits a flat extension, and that will readily imply that it admits a rank $\mathcal{M}(2)$-atomic (that is, 6 -atomic) representing measure. The $B(3)$ block in an extension $\mathcal{M}(3)$ can be generated by letting $\beta_{41}=\beta_{32}=\beta_{23}=\beta_{14}=u$, so that $B(3)$ can thus be written as

$$
\left(\begin{array}{cccc}
e+u & u & u & f+u \\
g+u & u & u & u \\
u & u & u & h+u \\
\beta_{50} & u & u & u \\
u & u & u & u \\
u & u & u & \beta_{05}
\end{array}\right) .
$$

As usual, let $W:=\mathcal{M}(2)^{-1} B(3)$ and let $C(3) \equiv\left(C_{i j}\right):=W^{*} \mathcal{M}(2) W$. Note that if $C(3)$ turns out to be Hankel, then $\mathcal{M}(3)$ is a flat extension of $\mathcal{M}(2)$. Since $C(3)$ is symmetric, to ensure that $C(3)$ is Hankel (and therefore $\mathcal{M}(3)$ is a moment matrix) we only need to solve the following system of equations:

$$
\left\{\begin{array}{l}
E_{1}:=C_{13}-C_{22}=0, \\
E_{2}:=C_{14}-C_{23}=0, \\
E_{3}:=C_{24}-C_{33}=0 .
\end{array}\right.
$$

This is a system of equations involving quadratic polynomials with 2 unknown variables (the new moments $\beta_{50}$ and $\beta_{05}$ ). A straightforward calculation shows that $E_{1}=0, E_{3}=0$, and that

$$
\begin{aligned}
E_{2}=0 \Longleftrightarrow & \left(c^{2}-a e\right)\left(d^{2}-b f\right) \beta_{50} \beta_{05} \\
& +\left(c^{2}-a e\right)\left(f^{3}-2 d f h+b h^{2}-d^{2} u+b f u\right) \beta_{50} \\
& +\left(d^{2}-b f\right)\left(e^{3}-2 c e g+a g^{2}-c^{2} u+a e u\right) \beta_{05} \\
& +\left(e^{3}-2 c e g+a g^{2}-c^{2} u+a e u\right)\left(f^{3}-2 d f h+b h^{2}-d^{2} u+b f u\right)=0 \\
\Longleftrightarrow & \kappa \lambda \beta_{50} \beta_{05}+\kappa \mu \beta_{50}+\lambda \nu \beta_{05}+\nu \mu=0,
\end{aligned}
$$

where $\kappa, \lambda, \mu$, and $\nu$ have the obvious definitions. If $\kappa, \lambda \neq 0$, then $\beta_{05}=\frac{-\mu \nu+\kappa \mu \beta_{50}}{\kappa \lambda \beta_{50}+\lambda \nu}$ (for $\beta_{50} \neq-\frac{\nu}{\kappa}$ ), which readily implies that $E_{2}=0$ admits infinitely many solutions. When $\kappa=0$ and $\lambda \neq 0$, we see that $E_{2}=\lambda \nu \beta_{05}+\mu \nu$, from which it follows that a solution always exists (and it is unique when $\nu \neq 0$ ). A similar argument shows that $\kappa \neq 0$ and $\lambda=0$ also yield a solution (which is unique when $\mu \neq 0$ ). We are thus left with the case when both $\kappa \equiv c^{2}-a e$ and $\lambda \equiv d^{2}-b f$ are equal to zero. Since $c$ and $d$ are in the diagonal of a positive semidefinite matrix, they must be positive. Thus, all of $a, b, e$, and $f$ are nonzero and we can set $e:=c^{2} / a$ and $f:=d^{2} / b$. In this case, the moment matrix is

$$
\mathcal{M}(2)=\left(\begin{array}{cccccc}
1+u & a+u & b+u & c+u & u & d+u \\
a+u & c+u & u & \frac{c^{2}}{a}+u & u & u \\
b+u & u & d+u & u & u & \frac{d^{2}}{b}+u \\
c+u & \frac{c^{2}}{a}+u & u & g+u & u & u \\
u & u & u & u & u & u \\
d+u & u & \frac{d^{2}}{b}+u & u & u & h+u
\end{array}\right) .
$$

Let $k:=\operatorname{det} \mathcal{M}(2) / \operatorname{det} \mathcal{M}(2)_{\{2,3,4,5,6\}}$. As in the proof of Proposition 3.2, we see that $k=\frac{-b^{2} c-a^{2} d+c d}{c d}>0$ and the first summand in the following decomposition of 
$\mathcal{M}(2)$ has rank 5 and is positive semidefinite (note that the $(1,1)$-entry is $1+u-k$ ):

$$
\begin{aligned}
& \mathcal{M}(2)=\left(\begin{array}{cccccc}
\frac{b^{2} c+a^{2} d+c d u}{c d} & a+u & b+u & c+u & u & d+u \\
a+u & c+u & u & \frac{c^{2}+a u}{a} & u & u \\
b+u & u & d+u & u & u & \frac{d^{2}+b u}{b} \\
c+u & \frac{c^{2}+a u}{a} & u & g+u & u & u \\
u & u & u & u & u & u \\
d+u & u & \frac{d^{2}+b u}{b} & u & u & h+u
\end{array}\right) \\
& +\left(\begin{array}{cccccc}
k & 0 & 0 & 0 & 0 & 0 \\
0 & 0 & 0 & 0 & 0 & 0 \\
0 & 0 & 0 & 0 & 0 & 0 \\
0 & 0 & 0 & 0 & 0 & 0 \\
0 & 0 & 0 & 0 & 0 & 0 \\
0 & 0 & 0 & 0 & 0 & 0
\end{array}\right) .
\end{aligned}
$$

The only column relation in the first summand is

$$
X Y=\frac{c d}{-b c-a d+c d} 1-\frac{a d}{-b c-a d+c d} X-\frac{b c}{-b c-a d+c d} Y=: \xi 1-\eta X-\theta Y
$$

Unless $\eta \theta=-\xi$, the conic that represents this column relation is a nondegenerate hyperbola, and therefore the moment sequence associated to the moment matrix has a 5-atomic measure, by Theorem 1.2. In the case when the conic in (4.2) is a pair of intersecting lines (i.e., $(x+\theta)(y+\eta)=0$ ), we must have $c=a$ or $d=b$.

Thus, the remaining two specific cases to cover are $\mathcal{M}(2)$ with $c=a$ or $d=b$. Since $\mathcal{M}(2)$ is invertible, for any $B(3)$ block we will be able to find $W$ such that $\mathcal{M}(2) W=B(3)$. We propose to use a $B(3)$ block with new moments $\beta_{32}=\beta_{23}=$ $\beta_{14}=0$, and to then extend $\mathcal{M}(2)$ to $\mathcal{M}(3)$ using Šmul'jan's Lemma, that is, we will define $C(3):=W^{*} B(3)$. The goal is to establish that $C(3)$ is a Hankel matrix, and that requires verification of (4.1). Before we begin our detailed analysis, we need to make a few observations.

Let $d_{i}$ denote the principal minor of $\mathcal{M}(2)$ for $i=1, \ldots, 6$; since $\mathcal{M}(2)$ is positive and invertible, we know that these minors are all positive. Then

$$
d_{5}=-\frac{\left(b^{2} c+a^{2} d-c d\right)\left(-c^{3}+a^{2} g\right) u}{a^{2}} \quad \text { and } \quad d_{6}=\frac{d_{5}\left(-d^{3}+b^{2} h\right)}{b^{2}},
$$

which implies

$$
\left(b^{2} c+a^{2} d-c d\right)\left(-c^{3}+a^{2} g\right)<0 \quad \text { and } \quad-d^{3}+b^{2} h>0 .
$$

Next, we use Mathematica to solve $E_{1}=0$ for $\beta_{50}$ and $E_{3}=0$ for $\beta_{05}$, and we obtain

$$
\begin{aligned}
& \beta_{50}=\frac{1}{a^{2} c\left(b^{2} c+a^{2} d-c d\right)\left(-d^{3}+b^{2} h\right) u}\left(\alpha_{11} \beta_{41}^{2}+\alpha_{12} \beta_{41}+\alpha_{13}\right), \\
& \beta_{05}=\frac{1}{b^{2} d\left(b^{2} c+a^{2} d-c d\right)\left(c^{3}-a^{2} g\right)}\left(\alpha_{21} \beta_{41}+\alpha_{22}\right),
\end{aligned}
$$

where the $\alpha_{i j}$ 's are polynomials in $a, b, c, d, g, h$, and $u$. Since $a, b \neq 0, c, d>0$, we can use (4.3) to show that both $\beta_{50}$ and $\beta_{05}$ above are well defined. We now substitute these values in $E_{2}$ and check that $E_{2}$ is a quadratic polynomial in $\beta_{41}$; indeed, we can readily show that the leading coefficient of $E_{2}$ is nonzero if $c=a$ or $d=b$. Thus, if the discriminant $\Delta$ of this quadratic polynomial is nonnegative, 
then (4.1) has at least one solution. We are now ready to deal with the two special cases: $c=a$ and $d=b$. If $c=a$, then

$$
\Delta=\frac{a^{2} u^{2}(a-g)^{2}\left(-d^{3}+b^{2} h\right)^{2} F_{1}(a, b, d, h)}{b^{4} d^{2}},
$$

where

$$
F_{1}(a, b, d, h)=(-1+a)^{2} b^{2} h^{2}+2 b^{2} d\left(2 b^{2}-3 d+3 a d\right) h-d^{4}\left(3 b^{2}-4 d+4 a d\right)
$$

is a concave upward quadratic polynomial in $h$. Notice that $\Delta \geq 0$ if and only if $F_{1} \geq 0$, which means that the discriminant of $F_{1}, \Delta_{1}:=16 b^{2} d^{2}\left(b^{2}-d+a d\right)^{3}$, needs to be zero or negative. In this case, we observe that

$$
\begin{aligned}
c & =a>0, \\
d_{3} & =-a b^{2}+a d-a^{2} d+a u-a^{2} u-b^{2} u+d u-a d u>0, \\
d_{4} & =-d_{3}(a-g)>0(\Rightarrow a-g<0), \\
d_{5} & =a\left(b^{2}-d+a d\right)(a-g) u>0,
\end{aligned}
$$

which leads to $b^{2}-d+a d<0$. Therefore, $\Delta_{1}<0$ and $\Delta>0$.

Similarly, if $d=b$, then

$$
\Delta=\frac{\left(-c^{3}+a^{2} g\right)^{2}(b-h)^{2} u^{2} F_{2}(a, b, c, h)}{a^{4}},
$$

where

$$
\begin{aligned}
F_{2}(a, b, d, h)= & (-1+b)^{2} h^{2} c^{2}+2 a(-1+b)\left(-2 b^{3}+3 b^{2} h+a h^{2}-b h^{2}\right) c \\
& +a^{2}\left(-4 a b^{3}+b^{4}+6 a b^{2} h-2 b^{3} h+a^{2} h^{2}-2 a b h^{2}+b^{2} h^{2}\right)
\end{aligned}
$$

is a concave upward quadratic polynomial in $c$. The discriminant of $F_{2}$ is $\Delta_{2}:=$ $16 a^{2}(-1+b)^{2} b^{3}(b-h)^{3}$; we observe that $d=b>0$ and $d_{6}=-d_{5}(b-h)>0$, which leads to $b-h<0$. Therefore, $\Delta_{2}<0$ and $\Delta>0$, which completes the proof.

\section{ACKNOWLEDGMENTs}

The authors are deeply grateful to the referee for many suggestions that led to significant improvements in the presentation. Many of the examples and portions of the proofs of some results in this paper were obtained using calculations with the software tool Mathematica Wol].

\section{REFERENCES}

[CuFi1] Raúl E. Curto and Lawrence A. Fialkow, Flat extensions of positive moment matrices: relations in analytic or conjugate terms, Nonselfadjoint operator algebras, operator theory, and related topics, Oper. Theory Adv. Appl., vol. 104, Birkhäuser, Basel, 1998, pp. 59-82. MR 1639649 (99i:47026)

[CuFi2] Raúl E. Curto and Lawrence A. Fialkow, Flat extensions of positive moment matrices: recursively generated relations, Mem. Amer. Math. Soc. 136 (1998), no. 648, x+56, DOI 10.1090/memo/0648. MR1445490 (99d:47015)

[CuFi3] Raúl E. Curto and Lawrence A. Fialkow, Solution of the singular quartic moment problem, J. Operator Theory 48 (2002), no. 2, 315-354. MR1938799 (2003j:47017)

[CuFi4] Raúl E. Curto and Lawrence A. Fialkow, Solution of the truncated hyperbolic moment problem, Integral Equations Operator Theory 52 (2005), no. 2, 181-218, DOI 10.1007/s00020-004-1340-6. MR2216081 (2007a:47017)

[Fia] Lawrence A. Fialkow, The truncated moment problem on parallel lines, Proc. 24th International Conference on Operator Theory, to appear. 
[FiNi] Lawrence Fialkow and Jiawang Nie, Positivity of Riesz functionals and solutions of quadratic and quartic moment problems, J. Funct. Anal. 258 (2010), no. 1, 328-356, DOI 10.1016/j.jfa.2009.09.015. MR2557966 (2010j:47017)

[Smu] Ju. L. Šmul'jan, An operator Hellinger integral (Russian), Mat. Sb. (N.S.) 49 (91) (1959), 381-430. MR0121662 (22 \#12396)

[Wol] Wolfram Research, Inc., Mathematica, Version 9.0, Wolfram Research, Inc., Champaign, IL, 2012.

Department of Mathematics, The University of Iowa, Iowa City, Iowa 52242

E-mail address: raul-curto@uiowa.edu

Department of Mathematics, Seoul National University, Seoul 151-742, Korea

Current address: Department of Mathematics, Inha University, Incheon 402-751, Korea

E-mail address: seyoo73@gmail.com 